\title{
Nature of the Quantum Potential
}

\author{
Anton Lipovka \\ Centro de Investigacion en Fisica, Universidad de Sonora, Sonora, Mexico \\ Email: aal@cifus.uson.mx \\ Received 16 April 2016; accepted 20 May 2016; published 23 May 2016 \\ Copyright (C) 2016 by author and Scientific Research Publishing Inc. \\ This work is licensed under the Creative Commons Attribution International License (CC BY). \\ http://creativecommons.org/licenses/by/4.0/ \\ (c) (i) Open Access
}

\begin{abstract}
In this paper we suggested a natural interpretation of the de Broglie-Bohm quantum potential, as the energy due to the oscillating electromagnetic field (virtual photon) coupled with moving charged particle. Generalization of the Schrödinger equation is obtained. The wave function is shown to be the eigenfunction of the Sturm-Liouville problem in which we expand virtual photon to include it implicitly into consideration. It is shown that the non-locality of quantum mechanics is related only with virtual photon. As an example, the zero-energy of harmonic oscillator is obtained from classical equations.
\end{abstract}

\section{Keywords}

Quantum Potential, de Broglie-Bohm Interpretation, Beables, Hidden Variables, the Meaning of the Wave Functions

\section{Introduction}

Interpretation of quantum theory (both classical and relativistic) since its birth to the present day is the subject of much debate on the fundaments of quantum physics. At first glance, the situation is reassuring, because now we have dozen different formulations of quantum theory [1] and can apply these powerful techniques for computing. However, there are a number of unresolved fundamental issues showing us that we do misunderstand something in the meaning of these formulations and we still do not have a final interpretation of quantum theory.

It is well known, the Bohmian formulation based on Louis de Broglie's pilot-wave theory suggests particularly a great conceptual advantage in possible interpretation because it is causal and not local. Finally it leads to the complete Hamilton function that contains the quantum potential, which reflects fundamental properties of nature (see [2]-[5] and references therein).

The quantum potential plays a central role in the formalism of Bohm and is used in modern physics and theoretical chemistry. It is of great importance because on the one hand the Bohmian formulation and quantum potential allows us to better understand the foundations, and fundamentals of theory. On the other hand quantum potential has multiples practical applications in different fields of knowledge (for example in the solid state 
physics, in theoretical chemistry etc.) because it gives us an opportunity to simulate different quantum effects without the involvement of the wave functions of system, and without solving the Schrödinger equation. In this case, the Monte Carlo method is applied to the hydrodynamic calculations, which are sometimes the only possibility to get the result, when the Schrödinger equation cannot be solved exactly.

Unfortunately up to now we did not understand clearly the nature of quantum potential and wave functions. This problem on the one hand provokes many speculations and strange hypothesis, but on the other hand this misunderstanding makes it difficult to progress in important areas such as quantum computers, solid state and many others. These fundamental questions were debated by many authors from the beginning of quantum theory. As an example we quote here the paper [1]: "Schrödinger invented this formulation in hope of casting quantum mechanics into a "congenial" and "intuitive" form-he was ultimately distressed when he found that his wavefunctions were functions in configuration space and did not actually exist out in ordinary three-dimensional space." and also: "The wavefunction should be regarded as a mathematical tool for calculating the outcomes of observations, not as a physically present entity existing in space...”

As it was mentioned above, this misunderstanding provokes many strange and exotic ideas and papers, where quantum potential is used for speculations, particularly in cosmology to construct the most intrigue entitycosmological constant. For this reasons it is of great importance to reveal physical sense and nature of quantum potential, and to determine the specific role of the wave function in the formalism of quantum mechanics.

Recently the paper [6] was published, in which a new foundation had been proposed to unify the quantum theory and relativity. In this paper it was shown that quantization naturally appeared as consequence of geometrical properties of our Universe, and Planck's constant was just adiabatic invariant of the electromagnetic field, determined by the Riemann-Cartan geometry (i.e. by the Hubble's and the cosmological constants). It was constructed self-consistent non-local quantum theory based on Einstein's generalized theory of gravity in the Riemann-Cartan manifold. It should be stressed that this theory does not requires any initial assumptions, external to the theory and alien by nature. So, we need not any axioms, wave functions or hidden variables.

Another paper in which a natural origin of quantization was suggested was published by Garcia-Morales [7]. In this work the quantization of the action $S$ was obtained as a consequence of thermodynamic theory (i.e. also without artificial axiomatic constructions).

These results also suggest that the orthodox formulations of quantum theory [1] based on the axiom of the wave function existence are not complete and should be reconsidered.

In present paper the quantum potential is shown to be formed by bounded electromagnetic field-virtual photon (see section 3 for details), which is a principle part and main participant for any bounded quantum system.

\section{Enigma of Quantum Potential}

Usually quantum potential in the Bohmian formulation of quantum theory is defined this way (we consider here one-particle case because that for many particles is treated the same manner). Schrödinger equation is

$$
i \hbar \frac{\partial \Psi(x, t)}{\partial t}=-\frac{\hbar^{2}}{2 m} \nabla^{2} \Psi(x, t)+U(x) \Psi(x, t) .
$$

Writing the wave function in form $\Psi(x, t)=R(x, t) \exp (i S(x, t) / \hbar)$ we immediately obtain two equations:

$$
\frac{\partial S}{\partial t}=-H_{t o t}=-\frac{(\nabla S)^{2}}{2 m}-U(x)+\frac{\hbar^{2}}{2 m} \frac{\nabla^{2} R}{R}
$$

and

$$
\frac{\partial R^{2}}{\partial t}+\nabla\left(R^{2} \frac{\nabla S}{m}\right)=0 .
$$

The first one is a Hamilton-Jacobi equation written for a modified Hamiltonian:

$$
H_{t o t}(t, x, p, R)=\frac{(\nabla S)^{2}}{2 m}+U(x)-Q(x, t)
$$

where $Q(x, t)=\frac{\hbar^{2}}{2 m} \frac{\nabla^{2} R}{R}$ is so-called quantum potential, and second one is a continuity equation written for 
$R^{2}$. This modified Hamiltonian usually named as $\Psi$-dependent one, and additional term (the quantum potential) usually interpreted as "an internal energy associated with a certain region of phase space, absent in classical mechanics, but arising in quantum mechanics from the uncertainty principle" [2]. It is difficult to agree with this point of view for many reasons. First of all note that the discussed system, described by Equation (1) is supposed to be isolated one, but it does not contains any variable electromagnetic field. Instead, it contains an artificial quantum potential.

It is clear, our system should contain an oscillating electromagnetic field produced by electron, but we do not see it in Equation (1). At the same time the Bohmian formulation has the following features:

- there is presence of hidden variables (it should be treated as a hint for presence of bounded electromagnetic field);

- it is causal (so, it should be a classical field theory);

- not local (presence of an electromagnetic field in theory).

Taking into account the fact that the only real substance we have in our arsenal to capture experimental events is electromagnetic field (this is an experimental fact), we can conclude that this field we lost in the beginning. This "lost" field was found in [6] and now we are able to identify quantum potential with that bounded non-local classical electromagnetic field mentioned above.

\section{Nature Does Not Have Hidden Variables}

Recently [8] the idea was suggested that quantum potential appears due to a "concealed" motion. Namely author show that in the case when our system consist of two parts, one of which is characterized by observable coordinates $q_{i}(t)$ and other one has "concealed" coordinates $Q_{i}(t)$ (here we use notations of author), the Hamilton function of such a system can be shown to contain quantum potential, which "may be regarded as the kinetic energy of additional concealed freedoms" [8]. But the Lagrangian used by author is incomplete (see Equation (1.1) in [8]), so to begin with we need to write correct action for our system.

Action of the system under consideration one can write as:

$$
S_{\text {tot }}=S_{0}+S_{\text {int }}+S_{e m} \text {. }
$$

Here $S_{0}$ is action for particle(s) without electromagnetic field,

$$
S_{\text {int }}=-\frac{1}{c^{2}} \int j^{\alpha}(x) A_{\alpha}(x) \mathrm{d}^{4} x
$$

is the interaction between electromagnetic field and charge(s), and

$$
S_{e m}=-\frac{1}{16 \pi c} \int F^{\alpha \beta} F_{\alpha \beta} \mathrm{d}^{4} x
$$

is action for electromagnetic field. It can be seen that both $S_{i n t}$ and $S_{e m}$ contains the field coordinates, so in this case we are speaking about a bounded photon coupled with electron. Such bounded photon is named as a virtual photon (see for details [9]) and in present paper this definition is used.

It should be stressed here, the Bohmian formulation is non-relativistic one by origin, so we may restrict our speculation by non-relativistic case. Let us consider the hydrogen atom as an example, where to simplicity sake we believe $m_{e} \ll m_{p}$. So, we can consider one-body equation, as it was made in the case of writing down the Equation (1). It is easy to show that introduction of second body will not change main properties of our result, but leads to small corrections which have well-understood nature, but are not significant for our present aim.

The classical energy equation for our reduced classical system $S_{0}$ (fixed proton and moving electron) is $H=p^{2} / 2 m+U(x)=E$. It is important emphasize again: in this equation there are no harmonic electromagnetic field, which must take place in real system due to electron oscillations. Canonical quantization procedure applied to this relation leads directly to the Schrödinger Equation (1) for a particle moving in a potential $U(x)$. However the oscillating electron does produce harmonic electromagnetic field $\varphi(k, x)$ (which appears in (6), (7)) and this harmonic function can be used to write Fourier-transformation of the energy equation on 4-coordinates $x$. This is the way in which electromagnetic field appears implicitly in the Schrödinger Equation (1) and Hamilton function transforms to the operator (see also [6]):

$$
\int \hat{H} \varphi \mathrm{d}^{4} x=i \hbar \int \frac{\partial}{\partial t} \varphi \mathrm{d}^{4} x .
$$


Here integration is carried out over 4-volume, and Hamiltonian $\hat{H}$ is operator of Liouville written for reduced (incomplete) non-relativistic system $\hat{H}=-\hbar^{2} \nabla^{2} / 2 m+U(x)$, which corresponds to the problem of Sturm-Liouville with eigenfunctions $\Psi_{n}(x, t)=\exp \left(i S_{n} / \hbar\right)$. These eigenfunctions, in turn, form complete basis and we can expand our virtual photon, and this way include it into consideration through the coefficients of expansion $R_{n}\left(p_{\alpha}\right)$ :

$$
\varphi\left(p_{\alpha}, x^{\alpha}\right)=\sum R_{n}\left(p_{\alpha}\right) \Psi_{n}\left(x^{\alpha}\right) .
$$

Here we sum only over $n$, and index $\alpha$ is just to mention the fact we are working with 4-vector $p_{\alpha}$ and $x^{\alpha}$ in the Minkowsky space. By substitute this in (8) we have

$$
\int \hat{H} \sum R_{n}\left(p_{\alpha}\right) \Psi_{n}\left(x^{\alpha}\right) \mathrm{d}^{4} x=i \hbar \int \frac{\partial}{\partial t} \sum R_{n}\left(p_{\alpha}\right) \Psi_{n}\left(x^{\alpha}\right) \mathrm{d}^{4} x .
$$

These expressions actually are complete "quantum” non-local (integrated over the photon volume) equations to describe our system in Minkowsky space, with clearly written non-local hidden variables (beables) of the virtual photon (which are the coefficients $R_{n}\left(p_{\alpha}\right)$ ). In general case we should integrate this equations over 4-volume. However if we are interested in non-relativistic Schrödinger equation, we can evaluate these integrals by taking into account relation $v_{e} \ll c$, so, the main part of each integral is contributed by small region in vicinity of the electron and integration can be carried out easily. If we are interested in the case when our system stay in a defined state $n$, we can write (10) as:

$$
\left[-\frac{\hbar^{2}}{2 m} \nabla^{2} R-\frac{i \hbar}{m} \nabla R \nabla S+\frac{R(\nabla S)^{2}}{2 m}-\frac{i \hbar}{2 m} R \nabla^{2} S+R U(x)\right]=i \hbar \frac{\partial R}{\partial t}-R \frac{\partial S}{\partial t} .
$$

From this relation we immediately obtain the Hamilton-Jacobi Equation (2) written for a complete Hamiltonian of our system (4), where "quantum potential" now has clear sense and must be attributed to presence of the virtual photon (electromagnetic "pilot-wave"). As to the continuity Equation (3) written for $R^{2}$, it must be interpreted as continuity equation for the square coefficients of the expansion (into complete set of the eigen functions of corresponding problem of Sturm-Liouville) of the virtual photon coupled with electron and moving with velocity of the electron $\boldsymbol{v}_{e}$. It is actually an analog for well known Poynting's theorem. It should be stressed here in this continuity equation the Planck constant in fact does not appears, because it is classical equation for coefficients of expansion of classical electromagnetic field coupled with electron:

$$
\frac{\partial \rho}{\partial t}+\nabla(J)=0
$$

where

$$
\begin{gathered}
\rho=R^{2}, \\
\boldsymbol{J}=R^{2} \frac{\nabla S}{m}=R^{2} \boldsymbol{v}_{e} .
\end{gathered}
$$

To conclude this part it should be useful to make some comments on the Hamilton-Jacobi Equation (2)

$$
\frac{\partial S}{\partial t}=-H_{\text {tot }}=-\frac{(\nabla S)^{2}}{2 m}-U(x)+\frac{\hbar^{2}}{2 m} \frac{\nabla^{2} R}{R} .
$$

Now, when the physical sense of "quantum potential" (as classical potential that corresponds to the oscillating electromagnetic field with energy $\hbar \omega$ ) became clear, we may definitely interpret limit $\hbar \omega \rightarrow 0$ as an hypothetical situation with absence of the virtual photon (the energy of the oscillating electromagnetic field $\varphi(k, x)$ is zero). In this case we obtain a classical system with classical Hamilton function $H=\frac{(\nabla S)^{2}}{2 m}+U(x)$ for our incomplete system.

\section{Discussion}

Very fundamental and at the same time useful example suggests harmonic oscillator. We have discussed it from 
this point of view before in [6], but it would be interesting to discuss it briefly in the context of the proposed interpretation of the quantum potential. It should be done the same way as it was made in previous part (see eq. (5)-(15)). The Hamiltonian written for harmonic oscillator is:

$$
H=-\frac{\hbar^{2}}{2 m} \nabla^{2}+\frac{1}{2} m \omega^{2} r^{2} .
$$

Substituting coherent state wave function $\Psi=\exp \left(-m \omega r^{2} / 2 \hbar\right)$ of corresponding problem of SturmLiouville into Equation (10), immediately give us quantum potential for harmonic oscillator:

$$
Q=\hbar \omega / 2
$$

which should be recognized as energy of virtual photon in zero-state of harmonic oscillator (remember here the frequency of electron oscillations is the same that has the virtual photon). So, the total Hamilton function for "quantum" (in reality classical) harmonic oscillator in ground state is

$$
H_{t o t}(t, x, p, R)=\frac{(\nabla S)^{2}}{2 m}+U(x)-\hbar \omega / 2
$$

with the oscillation frequency of electron (and virtual photon) $\omega$. It should be noted here, in the paper [2] there is an error in the expression for the quantum potential of harmonic oscillator, which we have corrected in Equations (17) and (18).

One can see again - the total Hamilton function corresponds to the complete mechanical system classical by nature which does not contain the hidden variables. And so called "quantum potential" appears due to the presence of virtual photon with frequency $\omega$, which forms a "quantum” zero-state energy $\hbar \omega / 2$. So, the quantum potential and hidden variables should be naturally identified with oscillating classical electromagnetic field (virtual photon) coupled with the moving electron.

In light of these results it becomes immediately obvious meaning of Bell's theorem, as a classical statement about the impossibility of motions with a speed faster than light in the framework of the relativistic theory.

\section{Conclusions}

Conclusions of our work can be formulated as follows:

1) The quantum potential is shown to be an additional energy, electromagnetic by origin, which appears due to the presence of coupled harmonic electromagnetic field (virtual photon).

2) The wave functions are shown to be just a complete basis of the Sturm-Liouville problem (written for reduced system with action $S_{0}$ ), in which the virtual photon is expanded to include it implicitly into Schrödinger equation.

3) It is stressed that the non-locality of quantum mechanics is related only with this virtual photon, namely with distribution of harmonic electromagnetic field in the system under consideration.

\section{References}

[1] Styer, D.F., Balkin, M.S., Becker, K.M., Burns, M.R., Dudley, C.E., Forth, S.T., Gaumer, J.S., Kramer, M.A., Oertel, D.C., Park, L.H., Rinkoski, M.T., Smith, C.T. and Wotherspoon, T.D. (2002) Nine Formulations of Quantum Mechanics. American Journal of Physics, 70, 288-297.

[2] Dennis, G., de Gosson, M.A. and Hiley, B.J. (2015) Bohm’s Quantum Potential as an Internal Energy. Physics Letters A, 379, 1224-1227.

[3] Riggs, P.J. (2008) Reflections on the deBroglie-Bohm Quantum Potential. Erkenntnis, 68, 21-39.

[4] Esposito, S. (1999) Photon Wave Mechanics: A De Broglie-Bohm Approach. Foundations of Physics Letters, 12, 533-545. http://dx.doi.org/10.1023/A:1021643108407

[5] Grössing, G. (2009) On the Thermodynamic Origin of the Quantum Potential. Physica A, 388, 811-823. http://dx.doi.org/10.1016/j.physa.2008.11.033

[6] Lipovka, A. (2014) Planck Constant as Adiabatic Invariant Characterized by Hubble’s and Cosmological Constants. Journal of Applied Mathematics and Physics, 2, 61-71. http://lanl.arxiv.org/abs/1401.2404 http://dx.doi.org/10.4236/jamp.2014.25009 
[7] Garcia-Morales, V. (2015) Quantum Mechanics and the Principle of Least Radix Economy. Foundations of Physics, 45, 295-332. http://dx.doi.org/10.1007/s10701-015-9865-X

[8] Holland, P. (2015) Quantum Potential Energy as Concealed Motion. Foundations of Physics, 45, $134-141$. http://dx.doi.org/10.1007/s10701-014-9852-7

[9] Ginzburg, V. (1987) Theoretical Physics and Astrophysics. Pergamon Press, Oxford. 